\title{
On Medial Representations
}

\author{
Gabriella Sanniti di Baja \\ Institute of Cybernetics "E. Caianiello", CNR \\ Via Campi Flegrei 34, 80078 Pozzuoli (Naples), Italy \\ g.sannitidibaja@cib.na.cnr.it
}

\begin{abstract}
During the last half century, medial representations of objects have been involved in many biological and physical theories, and computer scientists have designed suitable algorithms for their computation. Aim of this paper is to provide a short, non-comprehensive, survey of the most common medial representation systems.
\end{abstract}

Keywords: Medial Axis, Grassfire, Shock Graph, Voronoi Diagram, Skeleton.

\section{Introduction}

In the image processing literature, much attention has been devoted to the medial representation of objects, mirroring an analogous interest in the physical and biological fields. Most of the activity in the field of medial representation has been inspired by the work done by Blum [1] to define the medial locus of a $2 \mathrm{D}$ object by introducing the notion of symmetry point and a growth process. For a $2 \mathrm{D}$ object, a symmetry point is a point that is center of a disc, bitangent two distinct sections of the boundary of the object and entirely contained in the interior of the object. A symmetry point can be associated with the length of the radius of the corresponding disc. In turn, the disc can be built via a growing process that, starting from the symmetry point, incorporates all object's point whose distance from the symmetry point does not overcome the length of the radius associated to the symmetry point itself. The envelope of the discs coincides with the object and the medial representation of the object is the locus of the centers (called medial locus in the following), associated with the lengths of the corresponding radii. Besides introducing the medial locus representation scheme (generally referred to as the Medial Axis), Blum also studied its geometric properties and showed its usefulness in shape analysis [2].

Successively, the Medial Axis, initially suggested by Blum for 2D objects, was extended to higher dimensions and a number of alternative representation systems, still identifying the medial locus of an object, were introduced. One of these alternative schemes is provided by the Grassfire Transformation. This transformation detects as medial locus the set of quenching points, which are found in the positions where a fire, lit at time $t=0$ at all border points of a dry grass field and uniformly propagating towards the interior, extinguishes. Each quenching point can be associated with the instant of time at which the fire died out at the position of the quenching point itself. Another scheme defines the Medial Axis as the location of shocks in a partial differential equation of motion at constant speed in a direction initially normal to the object 
boundary (Shock Graphs [3-6]). Also the Voronoi Diagram can be seen as strictly related to the Medial Axis, if its computation is done with respect to an infinite number of elements on the boundary of the object (see [6-11] for both the 2D and the 3D cases). Finally, skeletonization methods, based on the detection of suitable anchor points in the distance transform of the object with respect to the background and on the iterated application of topology preserving removal operations, originate a skeleton that does not substantially differ from the Medial Axis (see, e.g., [12-16] for both the $2 \mathrm{D}$ and $3 \mathrm{D}$ cases).

Other representations, somehow related to the Medial Axis but not considered in this paper, are the Midpoint Locus, introduced in [17] and the Process Induced Symmetric Axis (PISA), introduced in [18,19]. The Midpoint Locus is obtained as the locus of points placed in the middle of the chords connecting the pairs of bitangent points. PISA is the locus derived by connecting each pair of bitangent points along the shortest geodesic path on the bitangent disc/ball and taking the associated point to be the center of this geodesic path.

Techniques for extracting the medial representation based on Voronoi Diagram, grassfire transformation and shock graphs mainly represent continuous methods, working with real coordinates of points, while skeletonization techniques mainly constitute discrete methods, where the object is stored as a collection of pixels/voxels and the resulting skeleton is a connected subset of such a collection of pixels/voxels.

Whichever is the approach to compute the medial representation, this is a graph in case of planar objects, while is a dimensionally heterogeneous entity consisting of $2 \mathrm{D}$ manifolds, called sheets or surface patches, and 1D manifolds, called curves, in case of 3D objects. Actually, a medial representation exclusively consisting of 1D manifolds is possible for 3D solid objects, i.e. objects rid of cavities.

Medial representations have a number of features, some of which offer at the same time advantages and disadvantages. A typical example is the possibility to decompose the medial representation into a set of elementary parts (i.e., parts that consist of unbranching arcs or un-intersecting surfaces). The advantage of such a decomposition is that an elementary part of the representation can be mapped with a part of the object. The disadvantage is that, due to the high sensitivity of the medial locus to even small scale geometric aspects of the object, not all its elementary parts do correspond to individually significant portions of the object.

Some of the features that should characterize a medial representation, even if some of them cannot be satisfied at the same time and others require suitable pre- and postprocessing, are the following:

- Dimensionality reduction: the dimensionality of the medial representation is lower than that of the object. For a 2D object, the medial representation is a 1D set, union of arcs and curves; for a 3D object, the medial representation is union of surfaces, arcs and curves; it can also be union of arcs and curves, for solid objects with tubular shape;

- Centrality: the medial representation is medially placed within the object. In the discrete space and in the presence of objects including parts whose thickness is expressed by an even number of object elements, medialness can be achieved only in an approximate manner. Think of a straight segment consisting of an even number of elements: any of two elements - or both elements - are centered within the segment; 
- Topological equivalence: the medial representation is topologically equivalent to the object. The same number of object components and holes (i.e., background components) characterizes a $2 \mathrm{D}$ object and its representation; the same number of object components, cavities and tunnels characterizes a 3D object and its representation;

- Robustness: minor changes in the object's shape must produce minor changes of the medial representation. To guarantee robustness, pre-/post-processing is generally necessary. For example, noisy holes of a 2D object have to be filled in before computing the medial representation. This would otherwise include a closed curve surrounding each noisy hole, due to the topological equivalence of the representation and the object. In turn, noisy protrusions along the boundary of the object generate peripheral elementary parts of the representation, which can be removed by adequate post-processing (pruning).

- Invariance: the medial representation does not depend on the position/orientation of the object in space. The use of the Euclidean metric to detect the symmetry points, or -at least- of a reasonably good approximation of the Euclidean metric, is necessary to satisfy this feature;

- Reversibility: the object can be recovered starting from its medial representation. This feature may be contradictory with respect to the dimensionality reduction property, requiring that the medial representation of a $2 \mathrm{D}(3 \mathrm{D})$ object is union of arcs and curves (surfaces, arcs and curves). Actually, complete recovery is possible only if, in correspondence of all object's regions with even thickness, the medial representation is nearly thin (i.e., 2-element thick). Moreover, only for objects having tubular shape, a reasonably good recovery is possible from the 1D medial representation;

- Abstraction: the medial representation represents the object in a concise manner, capturing only those shape aspects that are relevant for the task at hand, is sufficient to unambiguously describe the object, and, at least intuitively, resembles the object's shape.

With the above features, a medial representation provides rich information on the geometry of the represented object, provides a basis for a description at multiple spatial scales and can be used in various image analysis tasks, such as object recognition.

Many other features would be desirable, such as the uniqueness of the representation and a precise correspondence between elements of the representation and elements of the object, but these can be seldom satisfied satisfactorily.

\section{The Medial Axis Transform}

The medial axis transform defined by Blum, MAT, is the locus of symmetry points, i.e., the object points placed midway between two sections of the object boundary. Each point of the MAT is the center of a ball, which touches the object boundary in two different points and can be associated with its distance to the boundary, i.e., the radius of the ball. The object can be recovered by taking the union of the balls associated to MAT points. These balls partially overlap with each other, so that the MAT is 
not necessarily a minimal coding. In fact, objects can be reconstructed from proper subsets of their MATs (see [20]).

The continuous MAT of a planar object consists of lines, each of which corresponding to a region of the object. See Fig. 1, where the MAT is superimposed onto the object. Three balls, centered on symmetry points located at the end of a line, along a line and at the intersection of three lines, are shown. In particular, the MAT of a polygon consists of straight lines and parabolic arcs, and peripheral MAT branches start from the convex vertices of the polygon. In the discrete case, all objects are actually polygons approximating the corresponding continuous object and, depending on the metric used to detect symmetry points and - hence - on the shape of the balls associated with the symmetry points, peripheral branches of the MAT originate from sufficiently sharp contour convexities. The continuous MAT of an object in $\mathrm{R}^{3}$ consists of surfaces and lines. For a polyhedron, peripheral MAT surfaces originate from convex edges of the polyhedron. The discrete 3D MAT also consists of discrete surfaces and lines, originating from sufficiently sharp convexities along the object boundary.

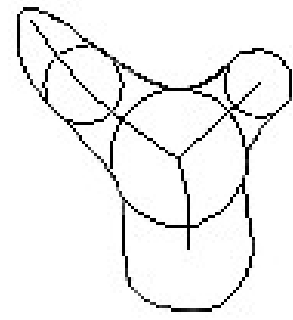

Fig. 1. The MAT of a $2 \mathrm{D}$ continuous object. Three maximal discs are shown.

The discrete MAT can be computed by detecting the centers of maximal balls in the distance transform of the object. In fact, a center of a maximal ball is a symmetry point, since the associated ball touches the boundary of the object in at least two distinct parts and is completely contained in the object.

Different discrete path-based distance functions can be used to compute the distance transform $[21,22]$ and the shape of the ball associated with the centers of maximal balls depends on the adopted metric. A quasi circular (spherical) shape characterizes only maximal balls computed by using quasi-Euclidean metrics, where suitable weights are used to properly differentiate the length of the unit moves from a pixel (voxel) to its edge- and vertex-neighbors (face-, edge- and vertex-neighbors). For metrics where equal weights are used for unit moves towards all neighbors, the MAT will not be stable under object rotation. See Fig. 2, where three 3D objects are shown to the left, while the sets of centers of maximal balls computed by using $\mathrm{D}^{6}$ (i.e., the $3 \mathrm{D}$ version of the $2 \mathrm{D}$ city-block distance) and $\mathrm{D}^{26}$ (i.e., the $3 \mathrm{D}$ version of the $2 \mathrm{D}$ chessboard distance) are respectively shown in the middle and to the right.

Another drawback of the discrete MAT is that since not all symmetry points result to be centers of maximal balls, the discrete MAT does not necessarily reflect the topology of the associated object. Again refer to Fig. 2, where only for the first object, a cube, the MAT consists of a single connected component when using $\mathrm{D}^{6}$ or $\mathrm{D}^{26}$. For 
the other two connected objects in Fig.2, the MAT includes more than one connected component or spurious tunnels. While invariance under rotation can be obtained by resorting to a quasi-Euclidean metric, topological equivalence between an object and its discrete MAT cannot be guaranteed.
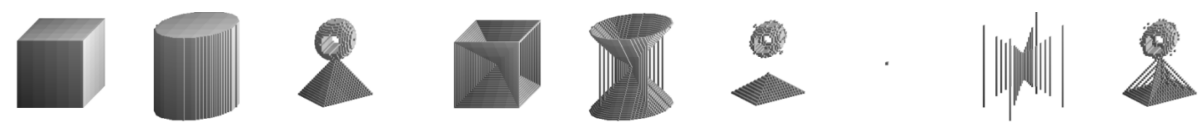

Fig. 2. Three 3D objects, left, their discrete MAT computed by detecting the centers of maximal balls on $\mathrm{D}^{6}$, middle, and on $\mathrm{D}^{26}$, right

To compute the MAT of a discrete surface in the 3D space, the distance transform to be used is the geodesic distance transform, where the object voxels delimiting the surface are used as the source from which distance information propagates towards the object voxels that are internal in the surface. In Fig. 3, the MAT of a rectangular object in upright and rotated position, as well as subjected to different bendings is shown superimposed on the surface. The metric adopted to compute the MAT is quasi-Euclidean. Thus, the MAT has the same structure whichever is the orientation of the object and independently of whether the surface is flat. For this object, the MAT also results to be topologically equivalent to the object.
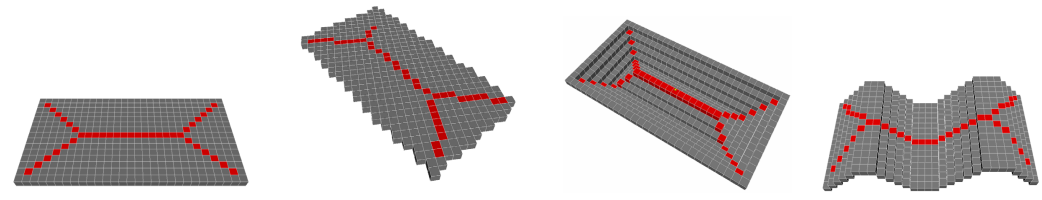

Fig. 3. The discrete MAT of a surface in the $3 \mathrm{D}$ space

\section{Grassfire and Shock Graph}

Grassfire Transformation and Shock Graph can be seen as a dynamic interpretation of the MAT concept. In both cases, the problem to be faced is the identification of singularities (quenching points and shocks) in a partial differential equation of motion, occurring at fixed speed in a direction initially perpendicular to the object boundary. The main difficulties concern numerical discretization of the flow and detection of singularities.

As concerns the grassfire, let us suppose that all points on the boundary of a dry grass field are set on fire at the same instant of time. In the absence of wind, the fire propagates at constant speed in every direction toward the innermost part of the field. Fire dies out at the quenching points, i.e., the points reached at the same instant of time by fire fronts originated at different parts of the boundary of the field. Of course, the quenching points are symmetrically placed within the field and the instant of time at which they are reached depends on their distance from the boundary of the field. Thus, the grassfire transform has the same properties as the MAT. 
An algorithm inspired by the grassfire process has been suggested, for example, to compute the straight skeleton of a polygon [23]. The wave-front propagation leading to the computation of the straight skeleton originates from consecutive subsets (the edges of the polygon) of the object boundary. These edges move towards the innermost part of the object, with constant speed and parallel to the initial edges of the polygon. While they move inwards, the edges change their lengths. The vertices of the polygon move along the bisectors, tracing the representation scheme, which consists of straight line segments only.

Two types of changes are possible for a moving edge: it may shrink to zero length (this event causes either the neighboring edges to become adjacent, if they have still positive length, or the wave-front to vanish); the edge splits due to interference (if self-interference occurs, two wave-fronts merge into a single one, otherwise splitting of a wave-front occurs).

The Shock Graph, [4], is obtained as the locus of singularities (shocks) generated during a propagation from the shape boundary. Each shock point is associated with a direction and with the (instantaneous) speed of flow [24]. Depending on the number of contact points and the flow direction, shock points are classified [25]: source and sink points determine the nodes of the graph, while the links connect source points to sink ones and define the arcs of the graph. Some attributes (intrinsic geometry of the region of the object that corresponds to a link, radius and flow direction of each node) are associated to the shock graph. The growing direction of the radius of the bitangent ball that is associated to each arc makes the shock graph representation somehow richer than the MAT.

Shock graphs are widely used for image matching, recognition and curve alignment. Most of the methods in the literature are mainly addressing the 2D case. However, shock graphs have also been extended to 3D. In the 3D case, the shock graph structure consists of components dimensionally heterogeneous and is not a planar graph.

\section{Voronoi Diagram}

In the continuous plane/space, given a finite set of points $S$, the Voronoi cell associated to a point $p$ of $S$ is defined as the subset of the plane/space with the points closer to $p$ than to any other point $q$ in $S$. Of course, for each point in the plane/space there exist at least one point of $S$ to which it is closer. In turn, a point can have the same distance from more than one point of $S$ and, if this is the case, the point belongs to a Voronoi edge separating two or more Voronoi cells. The union of the Voronoi cells of all points in $S$ is a partition of the plane/space called Voronoi Diagram of the set $S$. See Fig. 4 left.

If the set $S$ consists of a suitable number of points on the boundary of an object, the Voronoi diagram of $S$ contains edges extending outside of the object. The boundary of the object cuts the Voronoi diagram into an internal part and an external part. The internal part of the diagram is called the Voronoi skeleton. See Fig. 4 right. 

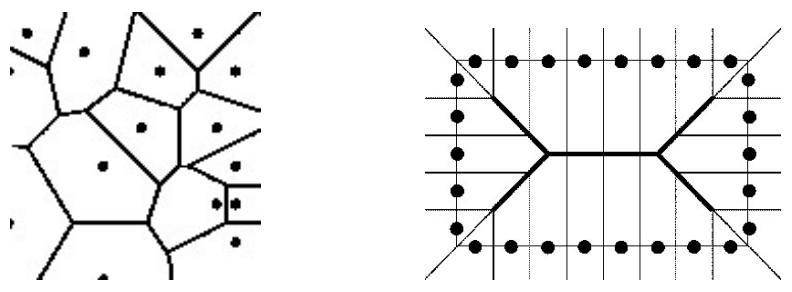

Fig. 4. The Voronoi diagram

The Voronoi diagram can be computed as the dual of the result obtained by computing the Delaunay triangulation. Given the set $S$, the Delaunay method triangulates the convex hull of $S$ in such a way that the circumcircle of each triangle includes only a generating point. The constituent elements of the Delaunay triangulation can be mapped onto the elements of the Voronoi diagram: the Delaunay points (i.e., the generating points of $S$ ) can be mapped to the Voronoi cells, and the Delaunay edges connect the points of $S$ which share a Voronoi edge.

In [26] it has been proved for the continuous case that if the density of the boundary points constituting $S$ goes to infinity, the Voronoi diagram of $S$ converges to the to the continuous medial locus, i.e., the skeleton of the object (once the Voronoi edges separating neighboring sampling points are disregarded). Actually, when the density increases, the number of Voronoi edges also increases and pruning has to be used in order to obtain a significant skeleton.

There is a very close relation between the Voronoi skeleton and the representations of the object obtained by using the medial axis transformation or the shocks of the grassfire flow, the only difference being that a disjoint set of points, obtained by sampling the boundary of the object, has to be used to build the Voronoi skeleton, instead of the continuous boundary as it is the case for the grassfire representation. As concerns the analogy with the MAT, we note that a point $p$ on a Voronoi edge is equidistant from two points on the boundary of the object. Thus, a circle of appropriate radius centered on $p$ has two points of contact with the boundary. In turn, if $p$ is at the intersection of Voronoi edges, $p$ is equidistant from at least three (generating) points on the boundary. Thus, a circle centered on $p$ has at least three points of contact with the boundary. As for the analogy with the grassfire, we note that if the fire is lit only on the points of a subset $S$ of the boundary, instead of on the whole boundary, the quenching points are the edges in the Voronoi diagram of $S$.

A key problem to be faced to extract the Voronoi skeleton of an object regards boundary sampling. A uniformly dense sampling produces a huge number of generating points in $S$ and, hence, a huge number of Voronoi edges, not all significant. Non uniform sampling that selects a larger number of generating points in regions of the boundary with high curvature could be a proper way to limit the number of non significant Voronoi edges produced by generating points located along straight portions of the boundary [27, 28]. However, non uniform sampling may cause a bias in the position of the main Voronoi edges and, hence a considerable deviation from the position expected for the skeleton. Therefore, uniform sampling is more often preferred and a pruning phase is added to the computation of the diagram to compute the Voronoi skeleton. 
To perform pruning of the Voronoi diagram and keep only the edges that are more stable and significant, significance measures have been introduced [29-32]. The significance measure can be used to establish a hierarchy of edges. The root of the hierarchy includes the most stable and significant edges, while edges that are farther and farther from the root have smaller and smaller significance. By pruning edges at different levels of the hierarchy, different approximations of the original object can be obtained, so originating a multi-scale representation. Significance measures can be local, e.g., the significance of each edge is evaluated by measuring the distance along the boundary of the two generating points from which the edge points are equidistant, or global, e.g., the significance of a group of edges is evaluated by comparing the shape of the objects recovered with and without the group of edges.

The extraction of the 3D Voronoi skeleton, e.g., [31,33], is definitely more complex than in 2D, due to the huge number of sampling points. Similarly to the $2 \mathrm{D}$ case, the methods are mainly based on the Delaunay tetrahedralization.

A way to reduce the computation load in the computation of both the $2 \mathrm{D}$ and the 3D Voronoi skeletons, could be to resort to an approximation of the Voronoi diagrams based on the discrete grid used for ridge extraction in distance maps [9].

\section{Skeletonization by DT}

The term skeletonization has been introduced in the framework of linear representation of 2D discrete objects and then extended to the 3D case. For a binary 2D image, skeletonization is a process changing the status of object pixels into that of background pixels until a subset of the object is obtained, which is a union of arcs and curves placed symmetrically with respect to the border of the object. For a 3D object, the general strategy of skeletonization is still the same: object voxels are changed to background voxels, provided that topology and geometry are not altered, until the skeleton is obtained. The 3D skeleton is either a set of 3D surfaces and curves (called surface skeleton) or, if reversibility is not requested and the object does not include cavities, it can consist only of 3D curves (called curve skeleton).

A skeletonization scheme, commonly followed in the literature, is iterative thinning. This is based on the repeated use of topology preserving removal operations with a local support (generally $3 \times 3$ in $2 \mathrm{D}$ and $3 \times 3 \times 3$ in $3 \mathrm{D}$ ). To guarantee skeleton medialness, removal operations are applied border after border, so that a long computation time, proportional to object thickness, is necessary to obtain the skeleton.

An alternative approach, characterized by a significantly smaller computational cost, is based on the use of the distance transform of the object. The distance transform is a labeled replica of the image, where the elements (pixel in 2D and voxels in $3 \mathrm{D}$ ) of one of the two sets (the object of interest $O I$ ) are labeled with the value of their distance from the other set (the reference set $R S$ ). The distance transform can be computed by means of a propagation process during which any object element $p$ receives distance information from its neighbors that either belong directly to $R S$ or are closer to $R S$ than $p$, and propagates distance information to its object neighbors that are farther than $p$ from $R S$. Thus, the distance transform can be seen as consisting of layers, each of which includes elements reached at the same instant of time by the propagating wave-front. When the sets $O I$ and $R S$ do not exhaust the image, the distance 
transform is generally called geodesic distance transform and the voxels that do not belong to either $O I$ or $R S$ are called barrier voxels. The structure of the distance transform is very much related to the structure of the grassfire transform, so making it appealing for computing the skeleton.

In the discrete space, the distance between two elements $p$ and $q$ is measured by the length of the shortest path linking $p$ to $q$. Depending on the kind of neighbors that can be used to build the linking path and on the weights used to measure the moves towards different kinds of neighbors, the computed distance will assume different values. In particular, proper selection of integer weights allows one to measure the length of the path in a way reasonably well approximating the Euclidean length of the path itself (see, [21,22]).

Skeletonization algorithms based on the use of distance transforms in 2D and 3D computed by different metrics are, for instance, [12-14, 16, 34].

The general scheme to compute the skeleton of a $2 \mathrm{D}$ object or the surface skeleton of a $3 \mathrm{D}$ object is the following. The layers of the distance transform are interpreted as the successive borders that would characterize the object, if this undergoes iterative skeletonization. Since all layers are directly available in the distance transform, most of the skeletal pixels/voxels can be directly identified and marked there in one inspection of the distance transform. These elements are the centers of the maximal balls (necessary to guarantee both medialness and reversibility of the skeleton), and the pixels/voxels placed in saddle configurations. Then, a small and fixed number of inspections is enough to identify and mark the remaining skeletal pixels/voxels necessary to obtain a skeleton homotopic to the object. Since the set of the centers of maximal balls is likely to be 2-element thick in correspondence of object regions whose thickness is expressed by an even number of elements, a suitable final thinning is performed to gain unit thickness. Finally, even if the distance transform based skeleton is definitely much less sensitive to noise than the Voronoi skeleton, pruning is generally taken into account to remove peripheral parts of the skeleton whose significance is negligible and whose presence in the skeleton would only make its structure more complex and heavy to use. At the end of the process, the skeleton of a 2D object is, as expected, a set union of arcs and curves, and the surface skeleton of a 3D object is a set union of intersecting surfaces (sheets) and curves (lines).

The surface skeleton can also be used to efficiently compute the curve skeleton of a 3D object, still by using distance information. In this case, the geodesic distance transform of the surface skeleton is necessary. To this aim, the voxels of the surface skeleton have to be classified so as to distinguish the voxels delimiting the surface skeleton (edge voxels), which will constitute the reference set $R S$, the voxels internal in the surface skeleton (internal voxels), which will constitute the object of interest $O I$, while all other voxels (background voxels, voxels belonging to lines and to intersection among surfaces or lines) will act as barrier voxels. The rationale for letting the intersections act as barriers is to guarantee that distance information propagated from the edge voxels delimiting a sheet does not flow through the intersections towards voxels internal in intersecting sheets. In this way, the centers of maximal balls found in the distance transform can be correctly interpreted as symmetry points of the various sheets forming the surface skeleton. Besides the centers of maximal balls, also the voxels classified as belonging to lines and to intersections carry relevant shape information and, as such, are marked as skeletal voxels. Once all these voxels have been 
identified and marked in the geodesic distance transform, the remaining voxels necessary to guarantee skeleton connectedness can be detected in a small number of inspections. Also in the case of curve skeletonization, final thinning and pruning are necessary to gain unit thickness and to obtain a skeleton consisting only of significant curves.

The best results are obtained if a quasi Euclidean metric is used to compute the distance transform. In Fig. 5 a) the skeleton of a 2D object, computed by weighting 3 and 4 the unit moves towards pixels sharing an edge and pixels sharing a vertex is shown superimposed on the input object. Fig 5 b), c) and d) respectively show a cylinder, its surface skeleton and its curve skeleton, obtained by using the weights 3,4 and 5 to measure the unit moves towards face-, edge- and vertex-neighbors, respectively.

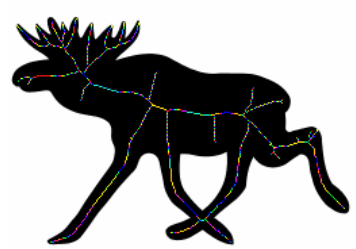

a)

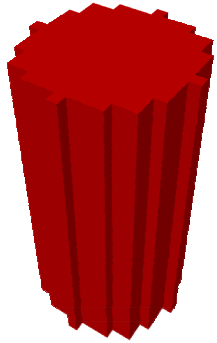

b)

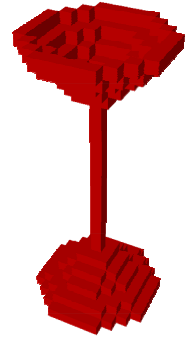

c)

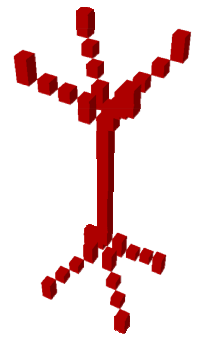

d)

Fig. 5. The skeleton of a 2D object,superimposed onto the object, a), a cylinder, b), its surface skeleton, c), and its curve skeleton, d), all computed by using a quasi Euclidean distance

\section{Applications of Medial Representations and Conclusion}

Some medial representation schemes have been briefly discussed in this paper, which are all related to the Medial Axis Transform. Medial representations have found many applications in computer vision and image analysis. A few examples, mainly concerning with the curve skeleton are given below to conclude this paper.

The curve skeleton has been used in virtual navigation to generate collision-free paths through a scene and, in particular, for virtual navigation through human organs. Typical examples are colonoscopy (e.g., [35]), bronchoscopy (e.g., [36]) and angioscopy (e.g., [37]). The above endoscopic methods are invasive and certainly uncomfortable for the patients. Since virtual endoscopy can produce images similar to those available by the traditional endoscopy without being invasive, the curve skeleton is a useful tool. After imaging, the skeleton of the object is computed and by moving a virtual camera through it, the inspection of the organ becomes possible.

The skeleton has also been used in computer graphics for the animation through vertex deformation of a static object. One of the problems is the difficulty in deforming vertices in the proximity of multiple limbs of the skeleton, still being able to maintain a surface with natural appearance. In [38], the weights associated with vertex deformation 
are computed automatically, by applying convolution to the medial axis/surface of the object, and fewer undesired artifacts appear in the animated surface.

Another application of the skeleton is for matching of 3D objects, where, given a query object, the task is to find similar objects in a database. If the curve-skeleton can differentiate the part structure of the original object, then also part matching is possible, by matching only parts of the objects against the query. For example, the method [39] uses graph matching techniques to match (parts of) the skeletons and to compare them. The skeletal graphs can be manually annotated to refine or restructure the search, so allowing to choose between a topological similarity and a geometric (shape) similarity. The matching results can also be used for driving a number of registration algorithms, most of which require a good initial guess to perform registration.

Finally, the skeleton has also been used in other image processing tasks like segmentation (e.g., [40]).

\section{References}

[1] Blum, H.: A transformation for extracting new descriptors of shape. MIT Press, Cambridge (1967)

[2] Blum, H., Nagel, R.: Shape Description Using Weighted Symmetric Axis Features. Pattern Recognition 10(3), 167-180 (1978)

[3] Kimia, B.B., Tannenbaum, A.R., Zucker, S.W.: Toward a Computational Theory of Shape: An Overview. In: Proceedings of the First European Conference on Computer Vision, Antibes, France, pp. 402-407. Springer, Heidelberg (1990)

[4] Kimia, B.B., Tannenbaum, A., Zucker, S.W.: Shape, Shocks, and Deformations I: The Components of Two-Dimensional Shape and the Reaction Diffusion Space. International Journal of Computer Vision 15, 189-224 (1995)

[5] Siddiqi, K., Kimia, B.B.: A Shock Grammar for Recognition. In: Proceedings of the Conference on Computer Vision and Pattern Recognition, pp. 507-513 (1996)

[6] Siddiqi, K., Shokoufandeh, A., Dickinson, S.J., Zucker, S.W.: Shock Graphs and Shape Matching. International Journal of Computer Vision 35(1), 13-32 (1999)

[7] Klein, F.: Vollständige Mittelachsenbeschreibung binärer Bildstrukturen mit euklidischer Metrik und korrekter Topologie. Ph.D. thesis, ETH Nr. 8441 (1987)

[8] Ogniewicz, R.: Discrete Voronoi Skeletons. Hartung-Gorre Verlag (1993)

[9] Arcelli, C., Sanniti di Baja, G.: Computing Voronoi diagrams in digital pictures. Pattern Recognition Letters 4(5), 383-389 (1986)

[10] Brandt, J., Algazi, V.: Continuous Skeleton Computation by Voronoi Diagram. Computer Vision Graphics Image Processing: Image Understanding 55(3), 329-338 (1992)

[11] Attali, D., Montanvert, A.: Semicontinuous skeletons of 2D and 3D shapes. In: Arcelli, C., Cordella, L.P., Sanniti di Baja, G. (eds.) Aspects of Visual Form Processing, pp. 32 41. World Scientific, Singapore (1994)

[12] Arcelli, C., Sanniti di Baja, G.: Euclidean skeleton via center-of-maximal-disc extraction. Image and Vision Computing 11, 163-173 (1993)

[13] Sanniti di Baja, G.: Well-shaped, stable and reversible skeletons from the (3,4)-distance transform. Visual Communication and Image Representation 5, 107-115 (1994)

[14] Sanniti di Baja, G., Thiel, E.: Skeletonization algorithm running on path-based distance maps. Image and Vision Computing 14, 47-57 (1997) 
[15] Svensson, S., Borgefors, G., Nyström, I.: On reversible skeletonization using anchorpoints from distance transforms. Journal of Visual Communication and Image Representation 10, 379-397 (1999)

[16] Sanniti di Baja, G., Svensson, S.: Surface skeletons detected on the D6 distance transform. In: Ferri, F.J., et al. (eds.) Advances in Pattern Recognition. LNCS, vol. 1121, pp. 387-396. Springer, Berlin (2000)

[17] Asada, H., Brady, M.: The Curvature Primal Sketch. IEEE Transactions on Pattern Analysis and Machine Intelligence 8, 2-14 (1983)

[18] Leyton, M.: A Process Grammar For Shape. Artificial Intelligence 34, 213-247 (1988)

[19] Leyton, M.: Inferring Causal History From Shape. Cognitive Science 13, 357-387 (1989)

[20] Borgefors, G., Nyström, I.: Efficient shape representation by minimizing the set of centers of maximal discs/spheres. Pattern Recognition Letters 18, 465-472 (1997)

[21] Borgefors, G.: Distance transformations in digital images. Computer Vision, Graphics, and Image Processing 34(3), 344-371 (1986)

[22] Borgefors, G.: On digital distance transform in three dimensions. Computer Vision and Image Understanding 64(3), 368-376 (1996)

[23] Aichholzer, O., Alberts, D., Aurenhammer, F., Gartner, B.: A novel type of skeleton for polygons. Journal of Universal Computer Science 1, 752-761 (1995)

[24] Giblin, P.J., Kimia, B.B.: On the local form and transitions of symmetry sets, medial axes, and shocks. International Journal of Computer Vision 54, 143-157 (2003)

[25] Giblin, P.J., Kimia, B.B.: A formal classification of 3D medial axis points and their local geometry. In: Proc. CVPR 2000, vol. I, pp. 566-575. IEEE Computer Society, Los Alamitos (2000)

[26] Schmitt, M.: Some examples of algorithms analysis in computational geometry by means of mathematical morphology techniques. In: Boissonnat, J.-D., Laumond, J.-P. (eds.) Geometry and Robotics. LNCS, vol. 391, pp. 225-246. Springer, Heidelberg (1989)

[27] Asada, H., Brady, M.: The curvature primal sketch. IEEE Trans. Pattern Analysis Machine Inteligence 8, 2-14 (1986)

[28] Weiss, I.: Shape Reconstruction on a Varying Mesh. IEEE Trans. Pattern Analysis Machine Inteligence 12(4), 345-362 (1990)

[29] Ogniewicz, R.L., Kübler, O.: Hierarchic Voronoi skeletons. Pattern Recognition 28(3), 343-359 (1995)

[30] Szekely, G.: Shape Characterization by Local Symmetries. Habilitationsschrift, Institut fur Kommunikationstechnik, Fachgruppe Bildwissenschaft, ETH Zurich (1996)

[31] Näf, M.: Voronoi Skeletons: a semicontinous implementation of the 'Symmetric Axis Transform' in 3D space, Ph.D. thesis, ETH Zurich, Commmunication Technology Institue, Image Analysis Group IKT/BIWI (1996)

[32] Katz, R., Pizer, S.M.: Untangling the Blum Medial Axis Transform. International Journal of Computer Vision 55(3), 139-153 (2003)

[33] Attali, D., Montanvert, A.: Computing and simplifying 2D and 3D continuous skeletons. Computer Vision and Image Understanding 67(3), 261-273 (1997)

[34] Arcelli, C., Sanniti di Baja, G., Serino, L.: From 3D discrete surface skeletons to curve skeletons. In: Campilho, A., Kamel, M. (eds.) ICIAR 2008. LNCS, vol. 5112, pp. 507516. Springer, Heidelberg (2008)

[35] Wan, M., Dachille, F., Kaufman, A.: Distance-Field Based Skeletons for Virtual Navigation. In: Proc. IEEE Visualization 2001, pp. 239-246 (2001)

[36] Perchet, D., Fetita, C.I., Preteux, F.: Advanced navigation tools for virtual bronchoscopy. In: Proc. SPIE Conf. on Image Processing: Algorithms and Systems III, vol. 5298, pp. $147-158$ (2004) 
[37] Bartz, D., Straßer, W., Skalej, M., Welte, D.: Interactive Exploration of Extra- and Intracranial Blood Vessels. In: Proc. 10th IEEE Visualization (VIS 1999), vol. 5 (1999)

[38] Bloomenthal, J.: Medial Based Vertex Deformation. In: Proc. SIGGRAPH/ Eurographics Symp. On Computer Animation, pp. 147-151 (2002)

[39] Sundar, H., Silver, D., Gagvani, N., Dickinson, S.: Skeleton based shape matching and retrieval. In: Proc. Shape Modelling and Applications Conference, SMI 2003, pp. 130-139 (2003)

[40] Pizer, S.M., Fritsch, D., Yushkevich, P., Johnson, V., Chaney, E.: Segmentation, Registration, and Measurement of Shape Variation via Image Object Shape. IEEE Trans. Medical Imaging 18, 851-865 (1999) 